\title{
Derivation of Kinetic Equations from the Generalized Langevin Equation*
}

\author{
A. Z. Akcasu and J. J. Duderstadt \\ Department of Nuclear Engineering, The University of Michigan, Ann Arbor, Michigan 48105 \\ (Received 28 April 1969)
}

\begin{abstract}
The projection operator techniques of Zwanzig and Mori are used to obtain a generalized Langevin equation describing the time evolution of the fluctuation of the microscopic phase density

$$
\delta g(\overrightarrow{\mathrm{x}}, \overrightarrow{\mathrm{p}}, t) \equiv g(\overrightarrow{\mathrm{x}}, \overrightarrow{\mathrm{p}}, t)-\langle g(\overrightarrow{\mathrm{x}}, \overrightarrow{\mathrm{p}}, t)\rangle
$$

for a classical many-particle system. This equation is then used to develop an exact kinetic equation for the time-correlation function $\left\langle\delta g(\overrightarrow{\mathrm{x}}, \overrightarrow{\mathrm{p}}, 0) \delta g\left(\overrightarrow{\mathrm{x}}^{\prime}, \overrightarrow{\mathrm{p}}^{\prime}, t\right)\right\rangle$ [which is the generalization of the Van Hove time-dependent pair correlation function $G(\overrightarrow{\mathrm{r}}, t)]$. In the lowest order of approximation, this kinetic description reduces to the Vlasov-like equation which has been used to study neutron scattering from liquids. A less restrictive approximation is obtained by utilizing weak-coupling perturbation theory to yield a generalized Fokker-Planck equation for the time-correlation function. Other possible approximation schemes are also discussed.
\end{abstract}

\section{INTRODUCTION}

A variety of techniques have been directed towards the calculation of time-correlation functions in nonequilibrium statistical mechanics. ${ }^{1-4} \mathrm{Re}-$ cently, there has been particular interest in the application of the projection operator techniques developed by Zwanzig ${ }^{5}$ for the study of irreversible processes. As one of several specific illustrations of projection methods, Zwanzig derived a generalized master equation for the autocorrelation function of a dynamical variable. This approach was subsequently generalized and extended by Mori, ${ }^{6}$ who utilized projection operators similar to those of Zwanzig to derive a generalized Langevin equation describing the time evolution of an arbitrary vector $\mathrm{A}(t)$ whose components are dynamical variables depending on the particle coordinates of a many-particle system. This equation was then used to study the corresponding correlation matrix $\left\langle\underline{\mathbf{A}}(t) \mathbf{A}^{*}(0)\right\rangle$, hence yielding a generalization of Zwānzig's work.

In this paper, our objective will be the calculation of the classical time-correlation function

$$
S\left(\overrightarrow{\mathrm{x}}, \overrightarrow{\mathrm{p}} ; \overrightarrow{\mathbf{x}}^{\prime}, \overrightarrow{\mathrm{p}}^{\prime}, t\right) \equiv\left\langle\delta g(\overrightarrow{\mathrm{x}}, \overrightarrow{\mathrm{p}}, 0) \delta g\left(\overrightarrow{\mathrm{x}}^{\prime}, \overrightarrow{\mathrm{p}}^{\prime}, t\right)\right\rangle,
$$

where $\delta g(\overrightarrow{\mathrm{x}}, \overrightarrow{\mathrm{p}}, t) \equiv g(\overrightarrow{\mathrm{x}}, \overrightarrow{\mathrm{p}}, t)-\langle g(\overrightarrow{\mathrm{x}}, \overrightarrow{\mathrm{p}}, t)\rangle$

is the fluctuation from equilibrium value of the microscopic phase density defined by

$$
g(\overrightarrow{\mathrm{x}}, \overrightarrow{\mathrm{p}}, t) \equiv \sum_{\alpha=1}^{N} \delta\left[\overrightarrow{\mathrm{x}}-\overrightarrow{\mathrm{x}}^{\alpha}(t)\right] \delta\left[\overrightarrow{\mathrm{p}}-\overrightarrow{\mathrm{p}}^{\alpha}(t)\right] .
$$

The time-correlation function $\mathcal{Q}\left(\overrightarrow{\mathrm{x}}, \overrightarrow{\mathrm{p}} ; \overrightarrow{\mathrm{x}}^{\prime}, \overrightarrow{\mathrm{p}}^{\prime}, t\right)$ is of particular interest in thermal neutron and light scattering from many-particle systems since its integral over $\overrightarrow{\mathrm{p}}$ and $\overrightarrow{\mathrm{p}}^{\prime}$ is just the Van Hove density correlation function ${ }^{7} G(\overrightarrow{\mathrm{r}}, t)$ which, in turn is directly related to the scattering cross section of the system under investigation. By applying the theory of Mori and Zwanzig, we will obtain a generalized Langevin equation for $\delta g(\overrightarrow{\mathrm{x}}, \overrightarrow{\mathrm{p}}, t)$ which can then be averaged to obtain an exact kinetic equation for the singlet density distribution function $f_{1}(\overrightarrow{\mathbf{x}}, \overrightarrow{\mathrm{p}}, t) \equiv\langle g(\overrightarrow{\mathrm{x}}, \overrightarrow{\mathrm{p}}, t)\rangle_{\rho}(0)$. However, of more direct interest is the related kinetic equation which describes the time evolution of the correlation function $\mathcal{S}\left(\overrightarrow{\mathrm{x}}, \overrightarrow{\mathrm{p}} ; \overrightarrow{\mathrm{x}}^{\prime}, \overrightarrow{\mathrm{p}}^{\prime}, t\right)$.

Recent theoretical investigations of neutron scattering in gases and liquids by Nelkin et al $.^{8}, 9$ have attempted to calculate $G(\vec{r}, t)$ by first obtaining and solving an approximate kinetic equation for $S\left(\overrightarrow{\mathrm{x}}, \overrightarrow{\mathrm{p}} ; \overrightarrow{\mathrm{x}}^{\prime}, \overrightarrow{\mathrm{p}}^{\prime}, t\right)$. By suitable approximations, we are able to reduce the exact kinetic equation to that studied by these authors. Furthermore, the exact kinetic equation for $s$ suggests less restrictive approximations which yield alternative kinetic descriptions of $\mathcal{S}[$ and hence of $G(\overrightarrow{\mathrm{r}}, t)]$ in a consistent fashion.

In the following work, we will first extend the generalized Langevin equation of Mori to "vectors" with continuous parameter dependence (in this case, upon $\overrightarrow{\mathrm{p}}$ ). This will lead to an exact equation describing the time evolution of $\delta g(\vec{x}, \vec{p}, t)$. Then by averaging this equation over a suitable ensemble, we can obtain a kinetic equation for the singlet density-distribution function $f_{1}(\overrightarrow{\mathrm{x}}, \overrightarrow{\mathrm{p}}, t)$. Following the work of Mori, we shall also derive an exact kinetic equation for the time correlation function $\mathrm{g}\left(\overrightarrow{\mathrm{x}}, \overrightarrow{\mathrm{p}} ; \overrightarrow{\mathrm{x}}^{\prime}, \overrightarrow{\mathrm{p}}^{\prime}, t\right)$ which will then be compared with the existing approximate kinetic descriptions of this quantity. As one possible consistent procedure for obtaining an approximate kinetic equation, we have calculated in some detail the weak- 
coupling limit of the exact kinetic equation using standard perturbative techniques.

\section{DERIVATION OF KINETIC EQUATION FOR $\left(\overrightarrow{\mathbf{x}}, \overrightarrow{\mathbf{p}} ; \overrightarrow{\mathbf{x}}^{\prime}, \overrightarrow{\mathbf{p}}^{\prime}, t\right)$}

Consider a vector $\mathrm{A}$ whose components $A_{j}(\Gamma)$ are dynamical variables of the phase $\Gamma \equiv\left(\vec{x}^{1}, \ldots, \overrightarrow{\mathrm{x}} N\right.$, $\left.\overrightarrow{\mathrm{p}}^{1}, \ldots, \overrightarrow{\mathrm{p}}^{N}\right)$ of a many-body system. Mori ${ }^{6}$ has demonstrated that the Louiville equation describing the time evolution of such a vector can be cast into the form of a generalized Langevin equation:

$$
\frac{d \underline{\mathrm{A}}}{d t}-i \underline{\Omega \mathrm{A}}(t)+\int_{0}^{t} d \tau \underline{\varphi}(\tau) \underline{\mathrm{A}}(t-\tau)=f(t),
$$

where the frequency matrix $\Omega$ is defined by

$$
i \underline{\Omega} \equiv\langle\underline{\dot{\mathrm{A}}}(0) \underline{\mathrm{A}} *(0)\rangle\langle\underline{\mathrm{A}}(0) \underline{\mathrm{A}} *(0)\rangle^{-1},
$$

the damping matrix $\underline{\varphi}$ is given by

$$
\underline{\varphi}(\tau) \equiv\langle\underline{\mathrm{f}}(\tau) \underline{\mathrm{f}} *(0)\rangle\langle\underline{\mathrm{A}}(0) \underline{\mathrm{A}} *(0)\rangle^{-1},
$$

and the random force $\underline{\mathrm{f}}(t)$ is given by

$$
\underline{\mathrm{f}}(t) \equiv e^{i t(1-P) L} i(1-P) L \underline{\mathrm{A}}(0) .
$$

Here, $\mathrm{A}^{*}$ is the row vector adjoint to $\mathrm{A} ; P$ is a projection operation defined by its action on an arbitrary dynamical variable $G$ as

$$
P \underline{G} \equiv\langle\underline{G} \underline{A} *(0)\rangle\langle\underline{A}(0) \underline{A} *(0)\rangle^{-1} \underline{A}(0) ;
$$

and \langle\rangle denotes an average over the equilibrium canonical ensemble $\rho_{0}(\Gamma)=e^{-\beta H / Z}, \beta \equiv 1 / k T$; and $L$ is the Liouville operator $L \equiv i\{H, \cdot\}$. It is a straightforward task to demonstrate ${ }^{6}$ that

$$
\langle\underline{\mathbf{f}}(t) \underline{\mathbf{A}} *(0)\rangle=0 \text {. }
$$

Hence, by postmultiplying (2) by $A^{*}(0)$ and averaging over $\rho_{0}(\Gamma)$, Mori was able to derive an exact equation for the correlation matrix

$$
\underline{\Gamma}(t) \equiv\langle\underline{\mathbf{A}}(t) \underline{\mathrm{A}} *(0)\rangle\langle\underline{\mathrm{A}}(0) \mathrm{A} *(0)\rangle^{-1},
$$

which took the form

$$
\frac{d \underline{\Gamma}}{d t}-i \underline{\Omega} \underline{\Gamma}(t)+\int_{0}^{t} d \tau \underline{\varphi}(\tau) \underline{\Gamma}(t-\tau)=0 .
$$

Mori's generalized Langevin equation (2) will now be used to study the time behavior of the dynamical variable $\delta g(\overrightarrow{\mathrm{x}}, \overrightarrow{\mathrm{p}}, t)$, or equivalently, its Fourier transform in space $\delta g(\overrightarrow{\mathrm{k}}, \overrightarrow{\mathrm{p}}, t)$. To this end, choose $\mathbf{A}(t)$ to be a "vector" whose "components" $A_{j} \rightarrow A(\overrightarrow{\mathrm{p}}, t)$ are indexed by a continuous parameter $\overrightarrow{\mathrm{p}}$ and defined by

$$
\begin{aligned}
A(\overrightarrow{\mathrm{p}}, t) \equiv & \delta g(\overrightarrow{\mathrm{k}}, \overrightarrow{\mathrm{p}}, t)=\sum_{\alpha=1}^{N} e^{i \overrightarrow{\mathrm{k}} \cdot \overrightarrow{\mathrm{x}}^{\alpha}(t)} \\
& \times \delta\left[\overrightarrow{\mathrm{p}}-\overrightarrow{\mathrm{p}}^{\alpha}(t)\right]-n \delta(\overrightarrow{\mathrm{k}}) M(\overrightarrow{\mathrm{p}}),
\end{aligned}
$$

where it has been noted that $\langle g(\overrightarrow{\mathrm{k}}, \overrightarrow{\mathrm{p}}, t)\rangle=n \delta(\overrightarrow{\mathrm{k}})$ $\times M(\overrightarrow{\mathrm{p}}), n$ being the equilibrium density, and $M(\overrightarrow{\mathrm{p}})=(\beta / 2 \pi m)^{3 / 2} \exp \left(-\beta p^{2} / 2 m\right)$.

The extension of the generalized Langevin equation to such a continuous representation is straightforward:

$$
\begin{aligned}
& \frac{\partial A}{\partial t}-i \int d \overrightarrow{\mathrm{p}}^{\prime} \Omega\left(\overrightarrow{\mathrm{p}}, \overrightarrow{\mathrm{p}}^{\prime}\right) A\left(\overrightarrow{\mathrm{p}}^{\prime}, t\right)+\int_{0}^{t} d \tau \\
& \quad \times \int d \overrightarrow{\mathrm{p}}^{\prime} \varphi\left(\overrightarrow{\mathrm{p}}, \overrightarrow{\mathrm{p}}^{\prime}, \tau\right) A\left(\overrightarrow{\mathrm{p}}^{\prime}, t-\tau\right)=f(\overrightarrow{\mathrm{p}}, t) .
\end{aligned}
$$

The appropriate projection operator (6) becomes $P G(\overrightarrow{\mathrm{p}}) \equiv \int d \overrightarrow{\mathrm{p}}^{\prime} \int d \overrightarrow{\mathrm{p}}^{\prime \prime}\left\langle G(\overrightarrow{\mathrm{p}}) A^{*}\left(\overrightarrow{\mathrm{p}}^{\prime}\right)\right\rangle \phi^{-1}\left(\overrightarrow{\mathrm{p}}^{\prime}, \overrightarrow{\mathrm{p}}^{\prime \prime}\right) A\left(\overrightarrow{\mathrm{p}}^{\prime \prime}\right)$,

where $\phi^{-1}\left(\vec{p}^{\prime}, \vec{p}^{\prime \prime}\right)$ is the inverse of the static correlation function defined by

$$
\begin{aligned}
\phi\left(\overrightarrow{\mathrm{p}}, \overrightarrow{\mathrm{p}}^{\prime}\right) \equiv & \left\langle A(\overrightarrow{\mathrm{p}}) A^{*}\left(\overrightarrow{\mathrm{p}}^{\prime}\right)\right\rangle=n \delta\left(\overrightarrow{\mathrm{p}}-\overrightarrow{\mathrm{p}}^{\prime}\right) M(\overrightarrow{\mathrm{p}}) \\
& +n^{2} M(\overrightarrow{\mathrm{p}}) M\left(\overrightarrow{\mathrm{p}}^{\prime}\right) h(k),
\end{aligned}
$$

where $h(k)$ is the Fourier transform of $[g(r)-1]$, $g(r)$ being the static pair correlation function. By its definition, $\phi^{-1}\left(\overrightarrow{\mathbf{p}}^{\prime}, \overrightarrow{\mathrm{p}}^{\prime \prime}\right)$ satisfies

$$
\int d \overrightarrow{\mathrm{p}}^{\prime} \phi\left(\overrightarrow{\mathrm{p}}, \overrightarrow{\mathrm{p}}^{\prime}\right) \phi^{-1}\left(\overrightarrow{\mathrm{p}}^{\prime}, \overrightarrow{\mathrm{p}}^{\prime \prime}\right)=\delta\left(\overrightarrow{\mathrm{p}}-\overrightarrow{\mathrm{p}}^{\prime \prime}\right) \text {. }
$$

But substituting the explicit form (13) into (14), we arrive at an inhomogeneous integral equation for $\phi^{-1}\left(\vec{p}, \vec{p}^{\prime \prime}\right)$

$n M(\overrightarrow{\mathrm{p}}) \phi^{-1}\left(\overrightarrow{\mathrm{p}}, \overrightarrow{\mathrm{p}}^{\prime \prime}\right)+n^{2} M(\overrightarrow{\mathrm{p}}) h(k)$

$$
\times \int d \overrightarrow{\mathrm{p}}^{\prime} M\left(\overrightarrow{\mathrm{p}}^{\prime}\right) \phi^{-1}\left(\overrightarrow{\mathrm{p}}^{\prime}, \overrightarrow{\mathrm{p}}^{\prime \prime}\right)=\delta\left(\overrightarrow{\mathrm{p}}-\overrightarrow{\mathrm{p}}^{\prime \prime}\right) .
$$

It is straightforward to demonstrate that the unique solution to Eq. (15) is

$$
\phi^{-1}\left(\overrightarrow{\mathrm{p}}^{\prime}, \overrightarrow{\mathrm{p}}^{\prime \prime}\right)=\delta\left(\overrightarrow{\mathrm{p}}^{\prime}-\overrightarrow{\mathrm{p}}^{\prime \prime}\right) / n M\left(\overrightarrow{\mathrm{p}}^{\prime}\right)-h(k) /[1+n h(k)] \text {. }
$$

Having obtained $\phi^{-1}\left(\overrightarrow{\mathrm{p}}^{\prime}, \overrightarrow{\mathrm{p}}^{\prime \prime}\right)$, we are now in a position to explicitly evaluate each of the terms in (11). First, note that the frequency kernel becomes

$$
i \Omega\left(\overrightarrow{\mathrm{p}}, \overrightarrow{\mathrm{p}}^{\prime}\right)=\int d \overrightarrow{\mathrm{p}}^{\prime \prime}\left\langle\dot{A}(\overrightarrow{\mathrm{p}}) A^{*}\left(\overrightarrow{\mathrm{p}}^{\prime \prime}\right)\right\rangle \phi^{-1}\left(\overrightarrow{\mathrm{p}}^{\prime \prime}, \overrightarrow{\mathrm{p}}^{\prime}\right) .
$$

But $\quad \dot{A}(\overrightarrow{\mathrm{p}})=\sum_{\alpha=1}^{N}\left(\frac{i \overrightarrow{\mathrm{k} \cdot \overrightarrow{\mathrm{p}}^{\alpha}}}{m}\right) e^{i \overrightarrow{\mathrm{k}} \cdot \overrightarrow{\mathrm{x}}^{\alpha}} \delta\left(\overrightarrow{\mathrm{p}}-\overrightarrow{\mathrm{p}}^{\alpha}\right)+\sigma(\overrightarrow{\mathrm{p}})$, 
where $\quad \sigma(\overrightarrow{\mathrm{p}}) \equiv \sum_{\alpha=1}^{N} e^{i \overrightarrow{\mathrm{k}} \cdot \overrightarrow{\mathrm{x}}^{\alpha}} \overrightarrow{\mathrm{F}}^{\alpha} \cdot \frac{\partial}{\partial \overrightarrow{\mathrm{p}}^{\alpha}} \delta\left(\overrightarrow{\mathrm{p}}-\overrightarrow{\mathrm{p}}^{\alpha}\right)$.

Hence, $\left\langle\dot{A}(\overrightarrow{\mathrm{p}}) A^{*}\left(\overrightarrow{\mathrm{p}}^{\prime \prime}\right)\right\rangle=(i \overrightarrow{\mathrm{k}} \cdot \overrightarrow{\mathrm{p}} / m) \phi\left(\overrightarrow{\mathrm{p}}, \overrightarrow{\mathrm{p}}^{\prime \prime}\right)$

$$
+\left\langle\sigma(\overrightarrow{\mathrm{p}}) A^{*}\left(\overrightarrow{\mathrm{p}}^{\prime \prime}\right)\right\rangle \text {. }
$$

To calculate $\left\langle\sigma(\overrightarrow{\mathrm{p}}) A^{*}\left(\overrightarrow{\mathrm{p}}^{\prime \prime}\right)\right\rangle$, note that

$$
\begin{aligned}
& \left\langle\sigma(\overrightarrow{\mathrm{p}}) A^{*}\left(\overrightarrow{\mathrm{p}}^{\prime \prime}\right)\right\rangle=\sum_{\alpha=1}^{N} \sum_{\beta=1}^{N} \\
& \times\left\langle e^{i \overrightarrow{\mathrm{k}} \cdot \overrightarrow{\mathrm{x}}^{\alpha}} \overrightarrow{\mathrm{F}}^{\alpha} \cdot \frac{\partial}{\partial \overrightarrow{\mathrm{p}}^{\alpha}} \delta\left(\overrightarrow{\mathrm{p}}-\overrightarrow{\mathrm{p}}^{\alpha}\right) e^{-i \overrightarrow{\mathrm{k}} \cdot \overrightarrow{\mathrm{x}}^{\beta}} \delta\left(\overrightarrow{\mathrm{p}}^{\prime \prime}-\overrightarrow{\mathrm{p}}^{\beta}\right)\right\rangle \\
& -\sum_{\alpha=1}^{N}\left\langle e^{i \overrightarrow{\mathrm{k}} \cdot \overrightarrow{\mathrm{x}}^{\alpha}} \overrightarrow{\mathrm{F}}^{\alpha} \cdot \frac{\partial}{\partial \overrightarrow{\mathrm{p}}^{\alpha}} \delta\left(\overrightarrow{\mathrm{p}}-\overrightarrow{\mathrm{p}}^{\alpha}\right)\right\rangle n \delta(\overrightarrow{\mathrm{k}}) M(\overrightarrow{\mathrm{p}}) .
\end{aligned}
$$

The second term vanishes since $\langle\vec{F} \alpha\rangle=0$. To evaluate the first term, define the microscopic current and density

$$
\overrightarrow{\mathrm{J}}_{k} \equiv \sum_{\alpha=1}^{N} \frac{\overrightarrow{\mathrm{p}}}{m} e^{i \overrightarrow{\mathrm{k}} \cdot \overrightarrow{\mathrm{x}}^{\alpha}} ; \quad \rho_{k} \equiv \sum_{\alpha=1}^{N} e^{i \overrightarrow{\mathrm{k}} \cdot \overrightarrow{\mathrm{x}}^{\alpha}} .
$$

Then (21) becomes

$$
\begin{aligned}
\left\langle\sigma(\overrightarrow{\mathrm{p}}) A^{*}\left(\overrightarrow{\mathrm{p}}^{\prime \prime}\right)\right\rangle= & {\left[-\frac{\partial}{\partial \overrightarrow{\mathrm{p}}} M(\overrightarrow{\mathrm{p}})\right] M\left(\overrightarrow{\mathrm{p}}^{\prime \prime}\right)\left\{m \left\langle\overrightarrow{\mathrm{J}}_{k}^{\rho}-k\right.\right.} \\
& \left.-i \overrightarrow{\mathrm{k}} \cdot \sum_{\alpha=1}^{N} \sum_{\beta=1}^{N}\left\langle\frac{\overrightarrow{\mathrm{p}}^{\alpha} \overrightarrow{\mathrm{p}}^{\alpha}}{m} e^{i \overrightarrow{\mathrm{k}} \cdot \overrightarrow{\mathrm{x}}^{\alpha \beta}}\right\rangle\right\} \\
= & -\frac{i \overrightarrow{\mathrm{k}} \cdot \overrightarrow{\mathrm{p}}}{m} M(\overrightarrow{\mathrm{p}}) M\left(\overrightarrow{\mathrm{p}}^{\prime \prime}\right) n^{2} g(k) .
\end{aligned}
$$

Hence, using (20) and (23) in (17), we find

$$
\begin{aligned}
& i \Omega\left(\mathrm{p}, \mathrm{p}^{\prime}\right)=\frac{i \overrightarrow{\mathrm{k}} \cdot \overrightarrow{\mathrm{p}}}{m} \int d \overrightarrow{\mathrm{p}}^{\prime \prime} \phi\left(\overrightarrow{\mathrm{p}}, \overrightarrow{\mathrm{p}}^{\prime \prime}\right) \phi^{-1}\left(\overrightarrow{\mathrm{p}}^{\prime \prime}, \overrightarrow{\mathrm{p}}^{\prime}\right) \\
&+\int d \overrightarrow{\mathrm{p}}{ }^{\prime \prime}\left[-\frac{i \overrightarrow{\mathrm{k}} \cdot \overrightarrow{\mathrm{p}}}{m} M(\overrightarrow{\mathrm{p}})\right] M\left(\overrightarrow{\mathrm{p}}^{\prime \prime} m^{2} g(k) \phi^{-1}\left(\overrightarrow{\mathrm{p}}^{\prime \prime}, \overrightarrow{\mathrm{p}}^{\prime}\right)\right. \\
&=\frac{i \overrightarrow{\mathrm{k}} \cdot \overrightarrow{\mathrm{p}}}{m} \delta\left(\overrightarrow{\mathrm{p}}-\overrightarrow{\mathrm{p}}^{\prime}\right)-\frac{i \overrightarrow{\mathrm{k}} \cdot \overrightarrow{\mathrm{p}}}{m} M(\overrightarrow{\mathrm{p}}) \frac{n g(k)}{1+n h(k)} .
\end{aligned}
$$

In a similar manner, the damping kernel $\varphi\left(\overrightarrow{\mathrm{p}}, \overrightarrow{\mathrm{p}}^{\prime}, t\right)$ becomes

$$
\begin{aligned}
\phi\left(\overrightarrow{\mathrm{p}}, \overrightarrow{\mathrm{p}}^{\prime}, t\right)= & \int d \overrightarrow{\mathrm{p}}{ }^{\prime \prime}\left\langle f(\overrightarrow{\mathrm{p}}, t) f^{*}\left(\overrightarrow{\mathrm{p}}^{\prime \prime}, 0\right)\right\rangle \phi^{-1}\left(\overrightarrow{\mathrm{p}}^{\prime \prime}, \overrightarrow{\mathrm{p}}^{\prime}\right) \\
= & \frac{\left\langle f(\overrightarrow{\mathrm{p}}, t) f^{*}\left(\overrightarrow{\mathrm{p}}^{\prime}, 0\right)\right\rangle}{n M\left(\overrightarrow{\mathrm{p}}^{\prime}\right)}-\frac{h(k)}{1+n h(k)} \\
& \times\left\langle f(\overrightarrow{\mathrm{p}}, t) \int d \overrightarrow{\mathrm{p}}^{\prime \prime} f^{*}\left(\overrightarrow{\mathrm{p}}^{\prime \prime}, 0\right)\right\rangle .
\end{aligned}
$$

Note that $f(\overrightarrow{\mathrm{p}}, 0)=i(l-P) L A(\overrightarrow{\mathrm{p}})=(l-P) \dot{A}(\overrightarrow{\mathrm{p}})$

$$
\begin{aligned}
& =(l-P) \sigma(\overrightarrow{\mathrm{p}})+\frac{i \overrightarrow{\mathrm{k}} \cdot \overrightarrow{\mathrm{p}}}{m}(l-P) A(\overrightarrow{\mathrm{p}}) \\
& =(l-P) \sigma(\overrightarrow{\mathrm{p}}) .
\end{aligned}
$$

Hence, the second term in (25) vanishes since $\int d \overrightarrow{\mathrm{p}}^{\prime \prime} \sigma\left(\mathrm{p}^{\prime \prime}\right)=0$. Furthermore,

$P \sigma(\overrightarrow{\mathrm{p}})=\int d \overrightarrow{\mathbf{p}}^{\prime} \int d \overrightarrow{\mathbf{p}}^{\prime \prime}\left\langle\sigma(\overrightarrow{\mathrm{p}}) A^{*}\left(\overrightarrow{\mathrm{p}}^{\prime}\right)\right\rangle \phi^{-1}\left(\overrightarrow{\mathrm{p}}^{\prime}, \overrightarrow{\mathrm{p}}^{\prime \prime}\right) A\left(\overrightarrow{\mathrm{p}}^{\prime \prime}\right)$

$$
\begin{aligned}
& =-\frac{\overrightarrow{\mathrm{k}} \cdot \overrightarrow{\mathrm{p}}}{m} M(\overrightarrow{\mathrm{p}}) \frac{n g(k)}{l+n h(k)} \int d \overrightarrow{\mathrm{p}}^{\prime \prime} A\left(\overrightarrow{\mathrm{p}}^{\prime \prime}\right) \\
& \equiv \mu(\overrightarrow{\mathrm{p}}) \int d \overrightarrow{\mathrm{p}}^{\prime \prime} A\left(\mathrm{p}^{\prime \prime}\right) ;
\end{aligned}
$$

but $\int d \overrightarrow{\mathrm{p}}^{\prime \prime} A\left(\overrightarrow{\mathrm{p}}^{\prime \prime}\right)=\rho_{k}-n \delta(\vec{k}) \equiv \delta \rho_{k}$,

so that (26) becomes

$$
f(\overrightarrow{\mathrm{p}}, 0)=\sigma(\overrightarrow{\mathrm{p}})-\mu(\overrightarrow{\mathrm{p}}) \delta \rho_{k} .
$$

Noting that

$$
\begin{aligned}
& \left\langle f(\overrightarrow{\mathrm{p}}, t) f *\left(\vec{p}^{\prime}, 0\right)\right\rangle=\left\langle\left[e^{i t(l-P) L} i(l-P) L A\right]\right. \\
& \left.\times\left[\delta^{*}\left(\overrightarrow{\mathrm{p}}^{\prime}\right)-\mu *\left(\overrightarrow{\mathrm{p}}^{\prime}\right) \delta \rho_{-k}\right]\right\rangle \\
& =\left\langle\left[e^{i t L(l-P)} i(l-P) L A\right]\right. \\
& \left.\times\left[(l-P) \sigma *_{-\mu} *(l-P) \delta \rho_{-k}\right]\right\rangle,
\end{aligned}
$$

and $(l-P) \delta \rho_{-k}=0$, our final form becomes

$\varphi\left(\overrightarrow{\mathrm{p}}, \overrightarrow{\mathrm{p}}^{\prime}, t\right)=\frac{\left\langle\sigma^{*}\left(\overrightarrow{\mathrm{p}}^{\prime}\right) e^{i t(l-P) L}\left[\sigma(\overrightarrow{\mathrm{p}})-\mu(\overrightarrow{\mathrm{p}}) \delta \rho_{k}\right]\right\rangle}{n M\left(\overrightarrow{\mathrm{p}}^{\prime}\right)}$.

To proceed further requires a detailed investigation of the propagator $\exp [i t(l-P) L]$. This will be deferred until Sec. III.

In summary then, the generalized Langevin equation for $A(\overrightarrow{\mathrm{p}}, t)=\delta g(\overrightarrow{\mathrm{k}}, \overrightarrow{\mathrm{p}}, t)$ can be written explicitly 


$$
\begin{aligned}
\frac{\partial}{\partial t} \delta g-\frac{i \overrightarrow{\mathrm{k}} \cdot \overrightarrow{\mathrm{p}}}{m} \delta g(\overrightarrow{\mathrm{k}}, \overrightarrow{\mathrm{p}}, t)+\frac{i \overrightarrow{\mathrm{k}} \cdot \overrightarrow{\mathrm{p}}}{m} M(\overrightarrow{\mathrm{p}}) \frac{n g(k)}{1+n h(k)} \\
\quad \times \int d \overrightarrow{\mathrm{p}} \delta g\left(\overrightarrow{\mathrm{k}}, \overrightarrow{\mathrm{p}}^{\prime}, t\right) \int_{0}^{t} d \tau \int d \overrightarrow{\mathrm{p}}^{\prime} \varphi \\
\quad \times\left(\overrightarrow{\mathrm{p}}, \overrightarrow{\mathrm{p}}^{\prime}, \tau\right) \delta g\left(\overrightarrow{\mathrm{k}}, \overrightarrow{\mathrm{p}}^{\prime}, t-\tau\right)=f(\overrightarrow{\mathrm{p}}, t) .
\end{aligned}
$$

To obtain a kinetic equation for the singlet phasedistribution function $f_{1}(\overrightarrow{\mathrm{k}}, \overrightarrow{\mathrm{p}}, t)$, one can follow Mori's prescription ${ }^{6}$ of averaging the generalized Langevin equation over a perturbed canonical ensemble

$$
\rho(0) \equiv e^{-\beta H-\overrightarrow{\mathrm{A}}^{*} \cdot \overrightarrow{\mathrm{B}}} / Z,
$$

where $B$ is the conjugate parameter which prescribes the initial values of $A$. By defining

$$
f_{1}(\overrightarrow{\mathrm{k}}, \overrightarrow{\mathrm{p}}, t) \equiv\langle g(\overrightarrow{\mathrm{k}}, \overrightarrow{\mathrm{p}}, t)\rangle_{\rho(0)},
$$

one obtains an exact equation for $f_{1}(\overrightarrow{\mathrm{k}}, \overrightarrow{\mathrm{p}}, t)$

$$
\begin{aligned}
\frac{\partial f_{1}}{\partial t}-\frac{i \overrightarrow{\mathrm{k}} \cdot \overrightarrow{\mathrm{p}}}{m} f_{1}(\overrightarrow{\mathrm{k}}, \overrightarrow{\mathrm{p}}, t)+\frac{i \overrightarrow{\mathrm{k}} \cdot \overrightarrow{\mathrm{p}}}{m} M(\overrightarrow{\mathrm{p}}) \frac{n g(k)}{1+n g(k)} \\
\quad \times \int d \overrightarrow{\mathrm{p}}^{\prime} f_{1}\left(\overrightarrow{\mathrm{k}}, \overrightarrow{\mathrm{p}}^{\prime}, t\right)+\int_{0}^{t} d \tau \int d \overrightarrow{\mathrm{p}}^{\prime} \\
\quad \times \varphi\left(\overrightarrow{\mathrm{p}}, \overrightarrow{\mathrm{p}}^{\prime}, \tau\right) f_{1}\left(\overrightarrow{\mathrm{k}}, \overrightarrow{\mathrm{p}}^{\prime}, t-\tau\right)=\langle f(\overrightarrow{\mathrm{p}}, t)\rangle_{\rho(0)} .
\end{aligned}
$$

In the linear approximation of small departures from equilibrium (small $B$ ),

$$
e^{-\beta H-A^{*} \cdot B} \sim e^{-\beta H}\left[1+A^{*} \cdot B\right]
$$

and the inhomogeneous term $\langle f(\overrightarrow{\mathrm{p}}, t)\rangle_{\rho}(0)$ vanishes. In the more general case of arbitrary departures from equilibrium, $\langle f(\overrightarrow{\mathrm{p}}, t)\rangle_{\rho(0)}$ will introduce nonlinear terms in $f_{1}(\overrightarrow{\mathrm{k}}, \overrightarrow{\mathrm{p}}, t)$, into the kinetic equation. However, our immediate concern is not with $f_{1}(\overrightarrow{\mathrm{k}}, \overrightarrow{\mathrm{p}}, t)$, but rather with the time-correlation function

$$
\Theta_{k}\left(\overrightarrow{\mathrm{p}}, \overrightarrow{\mathrm{p}}^{\prime \prime}, t\right) \equiv\left\langle\delta g^{*}\left(\overrightarrow{\mathrm{k}}, \overrightarrow{\mathrm{p}}^{\prime \prime}, 0\right) \delta g(\overrightarrow{\mathrm{k}}, \overrightarrow{\mathrm{p}}, t)\right\rangle .
$$

If we multiply (29) by $\delta g^{*}\left(\overrightarrow{\mathrm{k}}, \overrightarrow{\mathrm{p}}{ }^{\prime \prime}, 0\right)$ and then average over $\rho_{0}=e^{-\beta H} / Z$, recalling from (7),

$$
\left\langle f(\overrightarrow{\mathrm{p}}, t) \delta g^{*}\left(\overrightarrow{\mathrm{k}}, \overrightarrow{\mathrm{p}}^{\prime \prime}, 0\right)\right\rangle=0
$$

then we obtain an exact kinetic equation for

$$
\begin{aligned}
\frac{\partial g_{k}}{\partial t} & -\frac{i \overrightarrow{\mathrm{k}} \cdot \overrightarrow{\mathrm{p}}}{m} \Theta_{k}\left(\overrightarrow{\mathrm{p}}, \overrightarrow{\mathrm{p}}^{\prime \prime}, t\right)+\frac{i \overrightarrow{\mathrm{k}} \cdot \overrightarrow{\mathrm{p}}}{m} M(\overrightarrow{\mathrm{p}}) \frac{n g(k)}{1+n h(k)} \\
& \times \int d \overrightarrow{\mathrm{p}}^{\prime}{ }_{k}\left(\overrightarrow{\mathrm{p}}^{\prime}, \overrightarrow{\mathrm{p}}^{\prime \prime}, t\right)
\end{aligned}
$$

$$
=-\int_{0}^{t} d \tau \int d \overrightarrow{\mathrm{p}}^{\prime} \varphi\left(\overrightarrow{\mathrm{p}}, \overrightarrow{\mathrm{p}}^{\prime}, \tau\right){ }_{k}\left(\overrightarrow{\mathrm{p}}^{\prime}, \overrightarrow{\mathrm{p}}^{\prime \prime}, t-\tau\right),
$$

which is just the explicit generalization of Eq. (9) to continuous parameters. Hence, as has been suggested by other authors, ${ }^{10}$ we find that the timecorrelation function $S_{k}(\vec{p}, \vec{p} ", t)$ obeys a kinetic equation identical to the linearized kinetic equation for the singlet distribution function $f_{1}(\overrightarrow{\mathrm{k}}, \overrightarrow{\mathrm{p}}, t)$. It should again be stressed, however, that (32) is an exact equation for $\xi_{k}\left(\vec{p}, \vec{p}^{\prime \prime}, t\right)$ in which no linearization assumption was necessary. Of course, since (32) is exact, its solution is tantamount to solving the equations of motion themselves. Hence, (32) has only a formal significance until we introduce approximations sufficient to obtain an explicit and tractible form for the damping kernel $\varphi\left(\vec{p}, \vec{p}^{\prime}, \tau\right)$.

\section{APPROXIMATION OF DAMPING KERNEL}

One can generate approximate kinetic equations for $S_{k}\left(\overrightarrow{\mathrm{p}}, \overrightarrow{\mathrm{p}}^{\prime \prime}, t\right)$ in a consistent fashion by utilizing various approximations in the calculation of $\varphi\left(\overrightarrow{\mathrm{p}}, \overrightarrow{\mathrm{p}}^{\prime}, t\right)$. Of course, the general scheme of introducing approximations into such generalized master equations by approximating the behavior of the damping or memory term has been studied in some detail by Zwanzig ${ }^{5}$ and Mori. ${ }^{6}$ More recently, such an approach has proved to be remarkably successful in the study of simple time-correlation functions in liquids, ${ }^{3},{ }^{11}$ and hence might be expected to yield similarly good results in the study of more general phase-dependent time-correlation functions [ as well as in the derivation of approximate kinetic equations for $\left.f_{1}(\overrightarrow{\mathrm{k}}, \overrightarrow{\mathrm{p}}, t)\right]$.

There are several ways to approximate the damping kernel $\varphi\left(\vec{p}, \vec{p}^{\prime}, \tau\right)$. The crudest approximation would be to simply set

$$
\varphi\left(\overrightarrow{\mathrm{p}}, \overrightarrow{\mathrm{p}}^{\prime}, \tau\right) \equiv 0 .
$$

Interestingly enough, the resulting approximate kinetic equation

$$
\begin{gathered}
\frac{\partial S_{k}}{\partial t}-\frac{i \overrightarrow{\mathrm{k}} \cdot \overrightarrow{\mathrm{p}}}{m} \Theta_{k}\left(\overrightarrow{\mathrm{p}}, \overrightarrow{\mathrm{p}}^{\prime \prime}, t\right)+\frac{i \overrightarrow{\mathrm{k}} \cdot \overrightarrow{\mathrm{p}}}{m} M(\overrightarrow{\mathrm{p}}) \frac{n g(k)}{1+n h(k)} \\
\quad \times \int d \overrightarrow{\mathrm{p}}^{\prime} \Theta_{k}\left(\overrightarrow{\mathrm{p}}^{\prime}, \overrightarrow{\mathrm{p}}^{\prime \prime}, t\right)=0
\end{gathered}
$$

is just the Vlasov-like equation first derived by Zwanzig ${ }^{12}$ and later applied to the study of neutron scattering in liquids by Nelkin and Ranganathan. ${ }^{8}$

A natural generalization of this approximation of completely ignoring the damping term can be achieved by using a "two-component" kinetic description. That is, we choose a new vector $A(t)$ 
$=\operatorname{col}[a(\overrightarrow{\mathrm{p}}, t), b(\overrightarrow{\mathrm{p}}, t)]$ which has two continuous components

$$
\left.a(\overrightarrow{\mathrm{p}}, t) \equiv \delta g(\overrightarrow{\mathrm{k}}, \overrightarrow{\mathrm{p}}, t), \quad b(\overrightarrow{\mathrm{p}}, t) \equiv\left(l-P_{a}\right) \stackrel{\mathrm{a}}{(\mathrm{p}, t}\right),
$$

where $P_{a}$ is an explicit notation for the projection operator defined in (12). The second component is chosen such that (i) it is orthogonal to $a(\vec{p}, t)$, viz., $\left\langle a(\overrightarrow{\mathrm{p}}, t) b\left(\overrightarrow{\mathrm{p}}^{\prime}, 0\right)\right\rangle=0$, and (ii) $a(\overrightarrow{\mathrm{p}}, t)$ and $b(\overrightarrow{\mathrm{p}}, t)$ satisfy a conservation relation, viz. ,

$$
\begin{gathered}
\frac{\partial a}{\partial t}-\frac{i \overrightarrow{\mathrm{k}} \cdot \overrightarrow{\mathrm{p}}}{m} a(\overrightarrow{\mathrm{p}}, t)+\frac{i \overrightarrow{\mathrm{k}} \cdot \overrightarrow{\mathrm{p}}}{m} M(\overrightarrow{\mathrm{p}}) \frac{n g(k)}{1+n h(k)} \\
\times \int d \overrightarrow{\mathrm{p}}^{\prime} a\left(\overrightarrow{\mathrm{p}}^{\prime}, t\right)=b(\overrightarrow{\mathrm{p}}, t) .
\end{gathered}
$$

The latter is the first of the generalized Langevin equations in the two-component description as one can verify by a calculation similar to that outlined for the one-component description. [ We note that (36) does not contain any damping term or random force.] The second of the Langevin equations is obtained as

$$
\begin{aligned}
& \frac{\partial b}{\partial t}-i \int d \overrightarrow{\mathrm{p}}^{\prime} \Omega_{b}\left(\overrightarrow{\mathrm{p}}, \overrightarrow{\mathrm{p}}^{\prime}\right) b\left(\overrightarrow{\mathrm{p}}^{\prime}, t\right) \\
& +\int_{0}^{t} d \tau \int d \overrightarrow{\mathrm{p}}^{\prime} \varphi_{b}\left(\overrightarrow{\mathrm{p}}, \overrightarrow{\mathrm{p}}^{\prime}, \tau\right) b\left(\overrightarrow{\mathrm{p}}^{\prime}, t-\tau\right) \\
& \quad=-\int d \overrightarrow{\mathrm{p}}^{\prime}\left[\int d \overrightarrow{\mathrm{p}}^{\prime \prime}\left\langle b(\overrightarrow{\mathrm{p}}) b^{*}\left(\overrightarrow{\mathrm{p}}^{\prime \prime}\right)\right\rangle \phi_{a}^{-1}\left(\overrightarrow{\mathrm{p}}^{\prime \prime}, \overrightarrow{\mathrm{p}}^{\prime}\right)\right] a\left(\overrightarrow{\mathrm{p}}^{\prime}, t\right) \\
& \quad+f_{b}(\overrightarrow{\mathrm{p}}, t)
\end{aligned}
$$

where $i \Omega_{b}\left(\overrightarrow{\mathrm{p}}, \overrightarrow{\mathrm{p}}^{\prime}\right) \equiv \int d \overrightarrow{\mathrm{p}}^{\prime \prime}\left\langle\dot{b}(\overrightarrow{\mathrm{p}}) b^{*}\left(\overrightarrow{\mathrm{p}}^{\prime \prime}\right)\right\rangle \phi_{b}^{-1}\left(\overrightarrow{\mathrm{p}}^{\prime \prime}, \overrightarrow{\mathrm{p}}^{\prime}\right)$,

$$
\begin{gathered}
\varphi_{b}\left(\overrightarrow{\mathrm{p}}, \overrightarrow{\mathrm{p}}^{\prime}, \tau\right) \equiv \int d \overrightarrow{\mathrm{p}}^{\prime}\left\langle(l-P) \dot{b}^{*}\left(\overrightarrow{\mathrm{p}}^{\prime}\right) e^{i \tau(l-P) L}\right. \\
\times(l-P) \dot{b}(\overrightarrow{\mathrm{p}})\rangle \phi_{b}^{-1}\left(\overrightarrow{\mathrm{p}}^{\prime \prime}, \overrightarrow{\mathrm{p}}^{\prime}\right),
\end{gathered}
$$

and $\phi_{a}, b^{-1}\left(\overrightarrow{\mathrm{p}}^{\prime \prime}, \overrightarrow{\mathrm{p}}^{\prime}\right)$ is defined by Eq. (14) for $\phi_{a}\left(\overrightarrow{\mathrm{p}}, \overrightarrow{\mathrm{p}}^{\prime}\right) \equiv\left\langle a(\overrightarrow{\mathrm{p}}) a^{*}\left(\overrightarrow{\mathrm{p}}^{\prime}\right)\right\rangle$ and $\phi_{b}\left(\overrightarrow{\mathrm{p}}, \overrightarrow{\mathrm{p}}^{\prime}\right) \equiv\left\langle b(\overrightarrow{\mathrm{p}}) b^{*}\left(\overrightarrow{\mathrm{p}}^{\prime}\right)\right\rangle$. Consistent with out earlier approximation (33), we now approximate this exact set of equations by assuming

$$
\varphi_{b}\left(\overrightarrow{\mathrm{p}}, \overrightarrow{\mathrm{p}}^{\prime}, \tau\right) \equiv 0
$$

If we multiply through by $a^{*}\left(\vec{p}^{\prime \prime}\right)$ and average over the equilibrium ensemble $\rho_{0}$, then we find agener- alization of Eq. (34)

$$
\begin{aligned}
& \frac{\partial S_{k}}{\partial t}-\frac{i \overrightarrow{\mathrm{k}} \cdot \overrightarrow{\mathrm{p}}}{m} G_{k}\left(\overrightarrow{\mathrm{p}}, \overrightarrow{\mathrm{p}}^{\prime \prime}, t\right)+\frac{i \overrightarrow{\mathrm{k}} \cdot \overrightarrow{\mathrm{p}}}{m} M(\overrightarrow{\mathrm{p}}) \frac{n g(k)}{1+n h(k)} \\
& \times \int \overrightarrow{d \mathrm{p}^{\prime}} \mathcal{G}_{k}\left(\overrightarrow{\mathrm{p}}^{\prime}, \overrightarrow{\mathrm{p}}^{\prime \prime}, t\right)=\mathscr{H C}_{k}\left(\overrightarrow{\mathrm{p}}, \overrightarrow{\mathrm{p}}^{\prime \prime}, t\right), \\
& \frac{\partial \mathcal{K}}{\partial t}-i \int d \overrightarrow{\mathrm{p}}^{\prime} \Omega_{b}\left(\overrightarrow{\mathrm{p}}, \overrightarrow{\mathrm{p}}^{\prime}\right) \mathscr{H}_{k}\left(\overrightarrow{\mathrm{p}}^{\prime}, \overrightarrow{\mathrm{p}}^{\prime \prime}, t\right) \\
& =-\int \overrightarrow{d \mathrm{p}^{\prime}}\left[\int d \overrightarrow{\mathrm{p}}^{\prime \prime \prime}\left\langle b(\overrightarrow{\mathrm{p}}) b^{*}\left(\overrightarrow{\mathrm{p}}^{\prime \prime \prime}\right)\right\rangle \phi_{a}^{-1}\left(\overrightarrow{\mathrm{p}}^{\prime \prime \prime}, \overrightarrow{\mathrm{p}}^{\prime}\right)\right] \\
& \times S_{k}\left(\overrightarrow{\mathrm{p}}^{\prime}, \overrightarrow{\mathrm{p}}^{\prime \prime}, t\right),
\end{aligned}
$$

where we have defined. $\mathcal{H}_{k}\left(\overrightarrow{\mathrm{p}}, \overrightarrow{\mathrm{p}}^{\prime \prime}, t\right) \equiv\left\langle b(\overrightarrow{\mathrm{p}}, t) a^{*}\left(\overrightarrow{\mathrm{p}}^{\prime \prime}\right)\right\rangle$. Using the explicit forms for $a(\vec{p})$ and $b(\vec{p})$, one can easily calculate the kernels $\Omega_{b}\left(\overrightarrow{\mathrm{p}}, \overrightarrow{\mathrm{p}}^{\prime}\right)$ and $\left\langle b(\overrightarrow{\mathrm{p}}) b *\left(\overrightarrow{\mathrm{p}}^{\prime \prime}\right)\right\rangle$ appearing in (42). Hence, a twocomponent kinetic description has led to a system of two integrodifferential equations as the natural generalization of the Vlasov equation (34), which yield an approximate equation for $S_{k}\left(\overrightarrow{\mathrm{p}}, \overrightarrow{\mathrm{p}}^{\prime \prime}, t\right)$ if the cross correlation function $\mathfrak{H}_{k}\left(\overrightarrow{\mathrm{p}}, \overrightarrow{\mathrm{p}}^{\prime \prime}, t\right)$ is eliminated. Comparing (41) and (32), we find that $\mathcal{H}_{k}\left(\overrightarrow{\mathrm{p}}, \overrightarrow{\mathrm{p}}^{\prime \prime}, t\right)$ replaces the damping term in the latter. Hence, neglecting the damping term in the two-component description is equivalent to an approximate evaluation of the damping term in the one-component description, i.e., in (32).

A similar procedure could be used to generate still higher-order approximate kinetic equations by neglecting the damping term in an " $n$-component" kinetic description. Such a scheme is related to Mori's continued fraction representation ${ }^{13}$ of timecorrelation functions. However, such coupled sets of kinetic equations become rapidly unmanageable.

An alternative and perhaps more systematic approach to approximating $\varphi\left(\vec{p}, \vec{p}^{\prime}, t\right)$ consists of applying standard perturbative methods ${ }^{14},{ }^{15}$ directly to the form (28). That is, one can calculate $\varphi\left(\vec{p}, \vec{p}^{\prime}, t\right)$ to lowest order in a perturbation parameter [e.g., the density $n$ or the interaction strength $\lambda=O(V)]$. To illustrate this procedure, we shall apply a perturbation expansion in the coupling parameter $\lambda$ to obtain a Fokker-Plancklike form for the damping term. (A similar perturbation treatment in density should lead to a Boltzmann-like operator. )

We will first demonstrate that

$$
\left\langle f^{*}(O) e^{i t(l-P) L_{f}(0)}\right\rangle=\left\langle f^{*}(0) e^{i t L_{0}} f(0)\right\rangle+O\left(\lambda^{3}\right)
$$


where $\quad L_{0} \equiv-i \sum_{\alpha=1}^{N} \frac{\overrightarrow{\mathrm{p}}^{\alpha}}{m} \cdot \frac{\partial}{\partial \overrightarrow{\mathrm{x}}^{\alpha}}$.

To verify this, note that

$$
\begin{aligned}
i L P G= & \int d \overrightarrow{\mathrm{p}}^{\prime} \int d \overrightarrow{\mathrm{p}}^{\prime \prime}\left\langle G A^{*}\left(\overrightarrow{\mathrm{p}}^{\prime}\right)\right\rangle \phi^{-1}\left(\overrightarrow{\mathrm{p}}^{\prime}, \overrightarrow{\mathrm{p}}^{\prime \prime}\right) \\
& \times\left[\frac{i \overrightarrow{\mathrm{k}} \cdot \overrightarrow{\mathrm{p}}^{\prime \prime}}{m} A\left(\overrightarrow{\mathrm{p}}^{\prime \prime}\right)+\sigma\left(\overrightarrow{\mathrm{p}}^{\prime \prime}\right)\right], \\
i P L G= & \int d \overrightarrow{\mathrm{p}} \int d \overrightarrow{\mathrm{p}}^{\prime \prime}\left\langle G\left[\frac{i \overrightarrow{\mathrm{k}} \cdot \overrightarrow{\mathrm{p}}^{\prime}}{m} \cdot A^{*}\left(\overrightarrow{\mathrm{p}}^{\prime}\right)+\sigma^{*}\left(\overrightarrow{\mathrm{p}}^{\prime}\right)\right]\right\rangle \\
& \times \phi^{-1}\left(\overrightarrow{\mathrm{p}}^{\prime}, \overrightarrow{\mathrm{p}}^{\prime \prime}\right) A\left(\overrightarrow{\mathrm{p}}^{\prime \prime}\right) .
\end{aligned}
$$

Using Eq. (16) and noting $\phi^{-1}\left(\overrightarrow{\mathrm{p}}^{\prime}, \overrightarrow{\mathrm{p}}^{\prime \prime}\right)=\phi^{-1}\left(\overrightarrow{\mathrm{p}}^{\prime \prime}, \overrightarrow{\mathrm{p}}^{\prime}\right)$, we find

$$
\begin{aligned}
{[i L, P] G=} & \int d \overrightarrow{\mathrm{p}}^{\prime} \int d \overrightarrow{\mathrm{p}}^{\prime \prime}\left[n M\left(\overrightarrow{\mathrm{p}}^{\prime}\right)\right]^{-1} \\
& \times\left[\left\langle G A^{*}\left(\overrightarrow{\mathrm{p}}^{\prime}\right)\right\rangle \sigma\left(\overrightarrow{\mathrm{p}}^{\prime \prime}\right)-\left\langle G \sigma^{*}\left(\overrightarrow{\mathrm{p}}^{\prime}\right)\right\rangle A\left(\overrightarrow{\mathrm{p}}^{\prime \prime}\right)\right] \\
& +\left(\left\langle G \delta \rho_{k}^{*}\right\rangle \frac{i \overrightarrow{\mathrm{k}} \cdot \overrightarrow{\mathrm{J}}_{k}}{m}-\left\langle G \overrightarrow{\mathrm{J}}_{k}^{*}\right\rangle \cdot i \overrightarrow{\mathrm{k}} \rho_{k} / m\right) \\
& \times \frac{n g(k)}{1+n h(k)}=O(\lambda) .
\end{aligned}
$$

Hence, $\quad(l-P) L=L(l-P)+O(\lambda)$, and we find

$$
\begin{aligned}
e^{i t(l-P) L} f(0) & =e^{i t(l-P) L}(l-P) \sigma \\
& =e^{i t L_{0}} f(0)+O\left(\lambda^{2}\right) .
\end{aligned}
$$

Therefore, $\quad \varphi\left(\overrightarrow{\mathrm{p}}, \overrightarrow{\mathrm{p}}^{\prime}, t\right)=\left[n M\left(\overrightarrow{\mathrm{p}}^{\prime}\right)\right]^{-1}$

$$
\times\left\langle\sigma *\left(\overrightarrow{\mathrm{p}}^{\prime}\right) e^{i t L_{0}}\left[\sigma(\overrightarrow{\mathrm{p}})-\mu(\overrightarrow{\mathrm{p}}) \delta \rho_{k}\right]\right\rangle+O\left(\lambda^{3}\right)
$$

If we now recognuze that $e^{i t L_{0}}$ is an operator which merely displaces $\overrightarrow{\mathrm{x}} \alpha$ to $\overrightarrow{\mathrm{x}} \alpha+\overrightarrow{\mathrm{p}} \alpha t / m$, then we can calculate

$$
\begin{aligned}
& \left\langle\sigma^{*}\left(\overrightarrow{\mathrm{p}}^{\prime}\right) e^{\left.i t L_{0} \mu(\overrightarrow{\mathrm{p}}) \delta \rho_{k}\right\rangle}\right. \\
& =\mu(\overrightarrow{\mathrm{p}}) \sum_{\alpha=1}^{N} \sum_{\beta=1}^{N}\left\langle e^{-i \overrightarrow{\mathrm{k}} \cdot \overrightarrow{\mathrm{x}}^{\alpha}} \overrightarrow{\mathrm{F}}^{\alpha} \cdot \frac{\partial}{\partial \overrightarrow{\mathrm{p}}^{\alpha}} \delta\left(\overrightarrow{\mathrm{p}}^{\prime}-\overrightarrow{\mathrm{p}}^{\alpha}\right)\right. \\
& \times e^{\left.i \overrightarrow{\mathrm{k} \cdot \overrightarrow{\mathrm{x}}^{\beta}} e^{i \overrightarrow{\mathrm{k}} \cdot \overrightarrow{\mathrm{p}}^{\beta}} t / m\right\rangle}
\end{aligned}
$$$$
=-\left(\frac{i \overrightarrow{\mathrm{k}} \cdot \overrightarrow{\mathrm{p}}}{m} M(\overrightarrow{\mathrm{p}})\right)\left(\frac{i \overrightarrow{\mathrm{k}} \cdot \overrightarrow{\mathrm{p}}^{\prime}}{m} M\left(\overrightarrow{\mathrm{p}}^{\prime}\right)\right) \frac{n^{2} g^{2}(k)}{1+n h(k)}
$$$$
\times \exp \left(-k^{2} t^{2} / 2 m \beta\right) \text {. }
$$

Furthermore, $\left\langle\sigma^{*}\left(\overrightarrow{\mathrm{p}}^{\prime}\right) e^{i t L_{0}} \sigma(\overrightarrow{\mathrm{p}})\right\rangle$

$$
\begin{aligned}
& =\sum_{\alpha=1}^{N} \sum_{\beta=1}^{N}\left\langle e^{-i \overrightarrow{\mathrm{k}} \cdot \overrightarrow{\mathrm{x}}^{\alpha}} \overrightarrow{\mathrm{F}}^{\alpha} \cdot \frac{\partial}{\partial \overrightarrow{\mathrm{p}}^{\alpha}} \delta\left(\overrightarrow{\mathrm{p}}^{\prime}-\overrightarrow{\mathrm{p}}^{\alpha}\right) e^{i t L_{0} e^{i \overrightarrow{\mathrm{k}} \cdot \overrightarrow{\mathrm{x}}^{\beta}} \overrightarrow{\mathrm{F}}^{\beta}}\right. \\
& \left.\times \frac{\partial}{\partial \overrightarrow{\mathrm{p}}^{\beta}} \delta\left(\overrightarrow{\mathrm{p}}-\overrightarrow{\mathrm{p}}^{\beta}\right)\right\rangle=\sum_{\alpha=1}^{N} \sum_{\beta=1}^{N} \sum_{\gamma=1, \gamma \neq \beta}^{N}\left\langle e^{-i \overrightarrow{\mathrm{k}} \cdot \overrightarrow{\mathrm{x}}^{\alpha}} \overrightarrow{\mathrm{F}}^{\alpha}\right.
\end{aligned}
$$$$
\times \frac{\partial}{\partial_{\mathrm{p}}^{\alpha}} \delta\left(\overrightarrow{\mathrm{p}}^{\prime}-\overrightarrow{\mathrm{p}}^{\alpha}\right) e^{i \overrightarrow{\mathrm{k}} \cdot \overrightarrow{\mathrm{x}}^{\beta}} e^{i \overrightarrow{\mathrm{k}} \cdot \overrightarrow{\mathrm{p}} t / m_{\overrightarrow{\mathrm{F}}} \beta \gamma}
$$

$$
\left.\times\left(\overrightarrow{\mathbf{x}}^{\beta}-\overrightarrow{\mathbf{x}}^{\gamma}+\overrightarrow{\mathrm{p}}^{\beta} t / m-\overrightarrow{\mathrm{p}}^{\gamma} t / m\right) \cdot \frac{\partial}{\partial \overrightarrow{\mathrm{p}}^{\beta}} \delta\left(\overrightarrow{\mathrm{p}}-\overrightarrow{\mathrm{p}}^{\beta}\right)\right),
$$

or $\left\langle\sigma^{*}\left(\overrightarrow{\mathrm{p}^{\prime}}\right) e^{i t L_{0} \sigma(\mathrm{p})}\right\rangle=-\frac{\partial}{\partial \overrightarrow{\mathrm{p}}^{\prime}} M\left(\overrightarrow{\mathrm{p}}^{\prime}\right) e^{i \overrightarrow{\mathrm{k}} \cdot \overrightarrow{\mathrm{p}} t / m}$

$\times\left(\sum_{\substack{\alpha=1 \\ \alpha \neq \beta}}^{N} \sum_{\substack{\beta=1 \\ N}}^{N}\left\langle\overrightarrow{\mathrm{F}}^{\alpha} \overrightarrow{\mathrm{F}}^{\alpha \beta}\left(\overrightarrow{\mathrm{x}}^{\alpha}-\overrightarrow{\mathrm{x}}^{\beta}+\overrightarrow{\mathrm{p}}^{\prime} t / m-\overrightarrow{\mathrm{p}}^{\beta} t / m\right)\right\rangle\right)$

$\times \frac{\partial}{\partial \overrightarrow{\mathrm{p}}^{\prime}} \delta\left(\overrightarrow{\mathrm{p}}-\overrightarrow{\mathrm{p}}^{\prime}\right)-\frac{\partial}{\partial \overrightarrow{\mathrm{p}}^{\prime}} M\left(\overrightarrow{\mathrm{p}}^{\prime}\right) \frac{\partial}{\partial \overrightarrow{\mathrm{p}}} M(\overrightarrow{\mathrm{p}}) e^{i \overrightarrow{\mathrm{k}} \cdot \overrightarrow{\mathrm{p}} t / m}$

$$
\begin{aligned}
& :\left(\sum _ { \substack { \alpha = 1 \\
\alpha \neq \beta } } ^ { N } \sum _ { \substack { \alpha = 1 \\
\alpha \neq \beta } } ^ { N } \left\langle\overrightarrow{\mathrm{F}}^{\alpha} \overrightarrow{\mathrm{F}}^{\alpha \beta}\left(\overrightarrow{\mathrm{x}}^{\alpha}-\overrightarrow{\mathrm{x}}^{\beta}+\overrightarrow{\mathrm{p}}^{\prime} t / m-\overrightarrow{\mathrm{p}} t / m\right)\right.\right. \\
& \left.\left.\times e^{i \overrightarrow{\mathrm{k}} \cdot \overrightarrow{\mathrm{x}}^{\alpha \beta}}\right\rangle\right)+O\left(\lambda^{3}\right)
\end{aligned}
$$

Here we have noted that the terms arising from $\alpha \neq \beta \neq \gamma$ in Eq. (49b) are of $O\left(\lambda^{3}\right)$. Combining Eqs. (48) and (49b), one arrives at an expression for the damping kernel $\varphi\left(\overrightarrow{\mathrm{p}}, \overrightarrow{\mathrm{p}}^{\prime}, t\right)$ which is correct to $O\left(\lambda^{2}\right)$.

The damping term becomes particularly transparent in the Markovian limit in which we let $\lambda \rightarrow 0, t \rightarrow \infty, \vec{x} \rightarrow \infty$ in such a way that $\lambda^{2} t$ and $\lambda^{2} \vec{x}$ remain constant. (Note that both $t$ and $\overrightarrow{\mathrm{x}}$ refer to displacements in time and space in a stationary homogeneous medium.) Then 


$$
\begin{aligned}
& \int_{0}^{t} d \tau \int d \overrightarrow{\mathrm{p}}^{\prime} \varphi\left(\overrightarrow{\mathrm{p}}, \overrightarrow{\mathrm{p}}^{\prime}, \tau\right) \mathcal{S}_{k}\left(\overrightarrow{\mathrm{p}}^{\prime}, \overrightarrow{\mathrm{p}}^{\prime \prime}, t-\tau\right)-\int d \overrightarrow{\mathrm{p}}^{\prime} \\
& \times\left(\left.\int_{0}^{\infty} d \tau\left\langle\sigma^{*}\left(\overrightarrow{\mathrm{p}}^{\prime}\right) e^{i \tau L_{0} \sigma(\overrightarrow{\mathrm{p}})}\right\rangle\right|_{\overrightarrow{\mathrm{k}}=0}\right) \frac{S_{k}\left(\overrightarrow{\mathrm{p}}^{\prime}, \overrightarrow{\mathrm{p}}^{\prime \prime}, t\right)}{n M\left(\overrightarrow{\mathrm{p}}^{\prime}\right)} \\
& =-\int d \overrightarrow{\mathrm{p}}^{\prime}\left\{\frac { \partial } { \partial \vec { \mathrm { p } } ^ { \prime } } M ( \vec { \mathrm { p } } ^ { \prime } ) \cdot \left[\int _ { 0 } ^ { \infty } d \tau \sum _ { \alpha , \beta } ^ { \prime } \left\langle\overrightarrow{\mathrm{F}}^{\alpha}\right.\right.\right. \\
& \left.\left.\times \overrightarrow{\mathrm{F}}^{\alpha \beta}\left(\overrightarrow{\mathrm{x}}^{\alpha}-\overrightarrow{\mathrm{x}}^{\beta}+\frac{\overrightarrow{\mathrm{p}}^{\prime} \tau}{m}-\frac{\overrightarrow{\mathrm{p}}^{\beta} \tau}{m}\right)\right\rangle\right] \cdot \frac{\partial}{\partial \overrightarrow{\mathrm{p}}^{\prime}} \delta\left(\overrightarrow{\mathrm{p}}-\overrightarrow{\mathrm{p}}^{\prime}\right) \\
& +\frac{\partial}{\partial \overrightarrow{\mathrm{p}}^{\prime}} M\left(\overrightarrow{\mathrm{p}}^{\prime}\right) \frac{\partial}{\partial \overrightarrow{\mathrm{p}}} M(\overrightarrow{\mathrm{p}}):\left[\int _ { 0 } ^ { \infty } d \tau \sum _ { \alpha , \beta } ^ { \prime } \left\langle\overrightarrow{\mathrm{F}}^{\alpha} \overrightarrow{\mathrm{F}}^{\alpha \beta}\right.\right. \\
& \left.\left.\left.\times\left(\overrightarrow{\mathrm{x}}^{\alpha}-\overrightarrow{\mathrm{x}}^{\beta}+\frac{\overrightarrow{\mathrm{p}}^{\prime} \tau}{m}-\frac{\overrightarrow{\mathrm{p}} \tau}{m}\right)\right\rangle\right]\right\} \\
& \times \frac{\mathcal{G}_{k}\left(\overrightarrow{\mathrm{p}}^{\prime}, \overrightarrow{\mathrm{p}}^{\prime \prime}, t\right)}{n M\left(\overrightarrow{\mathrm{p}}^{\prime}\right)} \equiv J_{M}\left[\Theta_{k}\left(\overrightarrow{\mathrm{p}}^{\prime}, \overrightarrow{\mathrm{p}}^{\prime \prime}, t\right)\right] .
\end{aligned}
$$$$
=\beta \int \frac{d^{3} k}{(2 \pi)^{3}} \overrightarrow{\mathbf{k}} \tilde{V}(k) m S(k) \overrightarrow{\mathbf{k}} \tilde{V}(\mathbf{k}) \pi \delta\left(\overrightarrow{\mathbf{k}} \cdot \frac{\overrightarrow{\mathbf{p}}^{\prime}-\overrightarrow{\mathbf{p}}}{m}\right),
$$

and $S(k)$ is the static structure factor, $S(k)=1$ $+n h(k)$. Noting that

$$
\begin{aligned}
& \int d^{3} \overrightarrow{\mathrm{p}^{\prime}} \frac{\partial}{\partial \mathrm{p}} \cdot \vec{\xi}\left(\overrightarrow{\mathrm{p}^{\prime}}, \overrightarrow{\mathrm{p}}\right) \cdot\left(\overrightarrow{\mathrm{p}}-\overrightarrow{\mathrm{p}}^{\prime}\right) \\
& \quad \times\left[\psi\left(\overrightarrow{\mathrm{p}^{\prime}}, t\right) M(\overrightarrow{\mathrm{p}})-\psi(\overrightarrow{\mathrm{p}}, t) M\left(\overrightarrow{\mathrm{p}}^{\prime}\right)\right]=0,
\end{aligned}
$$

one can reduce (52) to a more familiar form:

$$
\begin{aligned}
& J_{M^{\prime}}[\psi]=\int d^{3} p^{\prime} \int \frac{d^{3} k}{8 \pi^{3}} n S(k) \tilde{V}(k) \overrightarrow{\mathrm{k}} \cdot \frac{\partial}{\partial \overrightarrow{\mathrm{p}}} \pi \delta\left(\overrightarrow{\mathrm{k}} \cdot \frac{\left(\overrightarrow{\mathrm{p}}-\overrightarrow{\mathrm{p}}^{\prime}\right)}{m}\right) \\
& \quad \times \tilde{V}(k) \overrightarrow{\mathbf{k}} \cdot\left(\frac{\partial}{\partial \mathrm{p}}-\frac{\partial}{\partial \overrightarrow{\mathrm{p}}^{\prime}}\right)\left[\psi\left(\overrightarrow{\mathrm{p}}^{\prime}, t\right) M(\overrightarrow{\mathrm{p}})+\psi(\overrightarrow{\mathrm{p}}, t) M\left(\overrightarrow{\mathrm{p}}^{\prime}\right)\right]
\end{aligned}
$$

In the absence of initial correlations, $S(k)=1$, and (55) reduces to the Fokker-Planck operator derived in the standard weak-coupling theory ${ }^{14-16}$ of manybody systems [e.g., see Eq. (6.18) of Ref. 14.]

Hence, the damping term (28) to lowest order in the interaction strength $\lambda$ can be regarded as a generalized Fokker-Planck operator which reduces to the more conventional form (55) in the Markovian limit.

In a similar manner, the damping term reduces to the linearized Boltzmann operator following a density expansion and subsequent Markovian limit $n \rightarrow 0, t \rightarrow \infty, \overrightarrow{\mathrm{x}} \rightarrow \infty$ such that $n t$ and $n \overrightarrow{\mathrm{x}}$ remain constant. ${ }^{17}$ (For an earlier derivation of the Boltzmann equation via projection techniques, see Mazur and Biel. ${ }^{18}$ )

\section{CONCLUSION}

In summary, we have utilized the projection operator techniques of Zwanzig and Mori to derive a kinetic equation for the time-correlation function of the microscopic phase density. When the damping term is neglected, this equation reduces to that considered in earlier studies of neutron scattering in liquids. A direct generalization of this particular approximation was given by using a twocomponent kinetic description of the many particle system. An alternative approximation scheme based upon standard perturbation theory was proposed and used to calculate the damping kernel directly. In the Markov limit, this approximation reduces to the linearized version of conventional kinetic equations (e.g., the Fokker-Planck equation). We feel that such equations are useful for studying neutron scattering in dense gases and liquids and can serve as a starting point for numer- 
ical calculations.

It is also possible to utilize this application of the generalized Langevin equation to study kinetic equations for the singlet distribution function itself. The principal approximations must then be introduced not only into the calculation of the damping kernel, but as well into the study of the inhomogeneous term $\langle f(\overrightarrow{\mathrm{p}}, t)\rangle_{\rho}(0)$.

It should be stressed that the generalized Langevin equation is an extremely general relation, and indeed is capable of describing the time evolution of any set of dynamical variables $A_{j}(t)$. The choice of an appropriate set of variables is not unique, and this choice will strongly influence the ease of calculation and accuracy of results obtained from approximations to the generalized Langevin equation. It is not at all obvious that the kinetic description of $\mathcal{G}_{k}\left(\mathrm{p}, \mathrm{p}^{\prime \prime}, t\right)$ will yield a more accurate calculation of the Van Hove function $G(k, t) \equiv\left\langle\rho_{k}(t)\right.$ $\left.\rho_{-k}(0)\right\rangle=\int d \overrightarrow{\mathrm{p}} \int d \overrightarrow{\mathrm{p}} " \prime \oint_{k}(\overrightarrow{\mathrm{p}}, \overrightarrow{\mathrm{p}} ", t)$ than would a direct calculation using a finite state vector $\mathrm{A} \equiv \operatorname{col}\left[\rho_{k}\right.$, $\left.\vec{J}_{k}, \vec{\Pi}_{k}\right]$ which contains the density as one of the components (hydrodynamic description. (The latter approach has been investigated by Akcasu and Daniels ${ }^{11}$ and was found to give very good agreement with molecular dynamics calculations by Rahman. ${ }^{19}$ Our effort in this work has been to merely indicate that such a kinetic description can be obtained rather directly from the generalized Langevin equation, and not to attempt to justify this level of description for a particular manybody system.

\section{ACKNOWLEDGMENTS}

This work has benefited very substantially from conversations with Professor Richard Osborn, Professor Noel Corngold, and Dr. Eugene Daniels.

\footnotetext{
*Work supported in part by the National Science Foundation under Contract No. GK-4853.

${ }^{1}$ K. Kawasaki and I. Oppenheim, Phys. Rev. 139, A1763 (1965)

${ }^{2}$ R. Desai and S. Yip, Phys. Rev. 166, 129 (1968).

${ }^{3}$ B. Berne, J. Boon, and S. A. Rice, J. Chem. Phys. 45, 1086 (1966).

${ }^{4}$ K. S. Singwi and A. Sjölander, Phys. Rev. 167, 152 (1968).

${ }^{5}$ R. Zwanzig, in Lectures in Theoretical Physics, edited by W. E. Brittin (John Wiley \& Sons, Inc., New York, 1961).

${ }^{6}$ H. Mori, Progr. Theoret. Phys. (Kyoto) 33,423 (1965).

${ }^{7}$ L. Van Hove, Phys. Rev. 95, 249 (1954).

${ }^{8}$ M. Nelkin and S. Ranganathan, Phys. Rev. 164, 222 (1967) .

${ }^{9} \mathrm{M}$. Nelkin and P. J. Ortoleva, in Neutron Inelastic Scattering (International Atomic Energy Agency, Vienna,
}

1968), paper SM-104/73.

${ }^{10} \mathrm{M}$. Nelkin, J. Van Leeuwen, and S. Yip, Neutron Inelastic Scattering (International Atomic Energy Agency, Vienna, 1964), Vol. II, p. 36.

${ }^{11}$ A. Z. Akcasu and G. Daniels, Bull. Am. Phys. Soc. 14,75 (1969).

${ }^{12}$ R. Zwanzig, Phys. Rev. 144, 170 (1966).

${ }^{13}$ H. Mori, Progr. Theoret. Phys. (Kyoto) 34, 399 (1965).

${ }^{14} \mathrm{P}$. Resibois, in $\mathrm{N}$ Particle Physics, edited by $\mathrm{E}$. Meeron (Gordon and Breach, Science Publishers, Inc., New York, 1967).

${ }^{15}$ R. Brout and I. Prigogine, Physica 22, 621 (1956).

${ }^{16}$ J. Ross, J. Chem. Phys. 24, 375 (1956).

${ }^{17} \mathrm{R}$. Zwanzig and M. Bixon (private communication).

${ }^{18} \mathrm{P}$. Mazur and J. Biel, Physica 32, 1633 (1966).

${ }^{19}$ A. Rahman, Neutron Inelastic Scattering (International Atomic Energy Agency, Vienna, 1968), Vol. I. 